Open Access

\title{
Coronary artery size and origin imaging in children: a comparative study of MRI and trans-thoracic echocardiography
}

Tarique Hussain ${ }^{1,2,3^{*}}$, Sujeev Mathur ${ }^{2}$, Sarah A. Peel ${ }^{1,2}$, Israel Valverde ${ }^{1,2}$, Karolina Bilska ${ }^{2}$, Markus Henningsson ${ }^{1}$, Rene M. Botnar ${ }^{1}$, John Simpson ${ }^{1,2}$ and Gerald F. Greil ${ }^{1,2,3}$

\begin{abstract}
Background: The purpose of this study was to see how coronary magnetic resonance angiography (CMRA) compared to echocardiography for the detection of coronary artery origins and to compare CMRA measurements for coronary dimensions in children with published echocardiographic reference values.

Methods: Enrolled patients underwent dual cardiac phase CMRA and echocardiography under the same anesthetic. Echocardiographic measurements of the right coronary artery (RCA), left anterior descending (LAD) and left main $(L M)$ were made. CMRA dimensions were assessed manually at the same points as the echocardiographic measurements. The number of proximal LAD branches imaged was also recorded in order to give an estimate of distal coronary tree visualization.

Results: Fifty patients (24 boys, mean age 4.0 years (range 18 days to 18 years)) underwent dual-phase CMRA. Coronary origins were identified in 47/50 cases for CMRA (remaining 3 were infants aged 3, 9 and 11 months). In comparison, origins were identified in $41 / 50$ cases for echo (remaining were all older children).

CMRA performed better than echocardiography in terms of distal visualization of the coronary tree (median 1 LAD branch vs. median $0 ; p=0.001$ ).

Bland-Altman plots show poor agreement between echocardiography and CMRA for coronary measurements. CMRA measurements did vary according to cardiac phase (systolic mean 1.90, s.d. $0.05 \mathrm{~mm}$ vs. diastolic mean 1.84, s.d. $0.05 \mathrm{~mm} ; p=0.002$ ).

Conclusions: Dual-phase CMRA has an excellent (94\%) success rate for the detection of coronary origins in children. Newborn infants remain challenging and echocardiography remains the accepted imaging modality for this age group. Echocardiographic reference ranges are not applicable to CMRA measurements as agreement was poor between modalities. Future coronary reference values, using any imaging modality, should quote the phase in which it was measured.
\end{abstract}

Keywords: Child, Infant, Newborn, Adolescent, Magnetic resonance imaging, Echocardiography, Coronary vessels

\footnotetext{
* Correspondence: tarique@doctors.org.uk

'Division of Imaging Sciences, King's College London, NIHR Biomedical

Research Centre at Guy's \& St Thomas' NHS Foundation Trust, London, UK

${ }^{2}$ Department of Paediatric Cardiology, Evelina London Children's Hospital,

Guy's \& St Thomas' NHS Foundation Trust, Westminster Bridge Road, London,

UK

Full list of author information is available at the end of the article
}

(c) 2015 Hussain et al. Open Access This article is distributed under the terms of the Creative Commons Attribution 4.0 International License (http://creativecommons.org/licenses/by/4.0/), which permits unrestricted use, distribution, and reproduction in any medium, provided you give appropriate credit to the original author(s) and the source, provide a link to the Creative Commons license, and indicate if changes were made. The Creative Commons Public Domain Dedication waiver (http://creativecommons.org/publicdomain/zero/1.0/) applies to the data made available in this article, unless otherwise stated. 


\section{Background}

In infants, it is appreciated that currently, echocardiography is the first line imaging method for delineation of the origin and course of the proximal portion of the coronary arteries [1]. However, echocardiographic imaging becomes progressively more difficult in children and adolescents due to patient size and poor transthoracic ultrasound windows.

Normal values exist for coronary dimensions using echocardiography, [2,3] but no such references exist for coronary magnetic resonance angiography (CMRA). The difficulty exists in producing normal MRI data is that young children and infants would require sedation or general anesthesia. This information, however, may be gained by cross-referencing MRI and echo data, so that MR dimensions can be validated systematically against echo-derived dimensions. This will be important for clinical settings, such as Kawasaki disease (or coronary allograft vasculopathy), where MRI is used for longitudinal follow up of coronary arteries.

In the setting of aneurysmal coronary segments and larger coronary arteries ( $>3 \mathrm{~mm}$ in adults), it has already been shown that CMRA is very accurate [4]. The purpose here is to be able to develop a reference for coronary dimensions in children to identify whether the segment in question is indeed dilated. This is particularly important for longitudinal follow-up and when echocardiographic windows are poor.

\section{Methods}

Institutional Review Board approval was obtained for this study (St. Thomas' research ethics committee reference 07/Q0704/3). The inclusion criterion in this prospective study was any patient undergoing a clinical cardiovascular MRI with 3d-whole heart acquisition under general anesthesia at our institution. Written, informed consent was obtained in each case from the patient (if older than 16 years) or from the legal guardian (if patient was younger). Enrolled patients underwent dual phase whole-heart 3D balanced steady state free precession (b-SSFP) imaging and echocardiography under general anesthesia [5].

\section{MRI}

Cardiac MRI was performed using a 1.5 Tesla Achieva clinical MR scanner (Philips Healthcare, Best, NL). The 3d whole heart approach, described by Beerbaum et al. [6] was implemented with noticeable changes. It has been recently demonstrated that dual-phase imaging (end-systole and mid-diastole) can improve coronary imaging by providing the ability to retrospectively select the optimum phase to be used for analysis [7]. Hence this approach was used for this study.
As with standard 3D SSFP sequences, the dual-phase sequence is respiratory-gated and ECG-triggered with a fat saturation pre-pulse to null fat signal and a $T_{2}$ prepulse to improve the myocardium to blood pool contrast [8]. Imaging was acquired in a sagittal orientation (repetition time (TR) /echo time (TE) $=3.4 / 1.7 \mathrm{~ms}$, flip angle $90^{\circ}, 60-120$ slices, isotropic resolution of $1-1.5 \mathrm{~mm}^{3}$, reconstructed resolution $0.5-0.75 \mathrm{~mm}^{3}$, acquisition window of $40-75 \mathrm{~ms})$. For children $15 \mathrm{~kg}$ or less, a twoelement coil was used (Flex M or Flex S). For children over $15 \mathrm{~kg}$, a five-element cardiac coil was used. Parallel imaging with a SENSitivity Encoding (SENSE) acceleration factor of 2 in the antero-posterior direction was also used [9]. Trigger delays were set for end-systole and mid-diastole. The cardiac rest periods were assessed with a high temporal resolution, balanced steady-state free precession (SSFP), four-chamber cine $(\mathrm{TR}=2.8$ $3.6 \mathrm{~ms}, \mathrm{TE}=1.4-1.8 \mathrm{~ms}$, no. of lines acquired per heart beat $=3$ to 20 , flip angle $60^{\circ}, 6$-mm-thick sections, 240 $300 \mathrm{~mm}$ field of view, $60-80$ phases). These parameters were adjusted accordingly to maintain temporal resolution with minimal interpolation. The dual-phase sequence employs navigators for each cardiac phase with a respiratory gating window of $3 \mathrm{~mm}$. Data is only accepted if both navigators, in any given cardiac cycle, fall within this gating window.

In addition, it has been shown that an automated programme is capable of more accurate definitions of cardiac rest periods than visual inspection [10]. It was therefore hoped that clearer definitions for cardiac restperiods would produce a similar effect. The middiastolic period was taken from cessation of movement of RCA (i.e., pause in visible filling of RV) to the beginning of atrial systole. This stringent definition covers both RCA and LAD diastolic rest periods [11]. The endsystolic period was taken from cessation of movement of the RCA (corresponding to lowest RV volume) to just before the beginning of opening of the tricuspid valve. The acquisition window was set according to the shortest rest period. Coronary dimensions were assessed manually at the same points as the echocardiographic measurements. The number of proximal LAD branches imaged was also recorded in order to give an estimate of distal coronary tree visualization.

Coronary origins were classified as abnormal if both echo \& MRI observers agreed, without doubt, that they were abnormal. In the case where modalities disagreed, reference was made either to earlier diagnostic catheterization or to previous surgical notes.

\section{Echocardiography}

Comprehensive echocardiographic studies were performed using the Philips IE33 ultrasound system (Philips Inc., Andover, MA, USA). 2-Dimensional echocardiography was 
performed using age appropriate probes (S5-1, S8-3, S124). The highest frequency probe yielding adequate depth penetration was employed. The Washington protocol for coronary dimensions was followed [3]. This protocol was chosen in preference as the measurement process is clearly defined. Images of the proximal coronary arteries (right coronary artery (RCA), left mainstem (LM) and left anterior descending (LAD)) were recorded as digital cines using short axis (in preference but alternatively, modified superiorly-tilted long-axis view for LAD was used). Operator assessment of coronary origins was recorded and stored cines were used to assess coronary dimensions.

Measurements of the RCA, LAD and LM were made from inner edge to inner edge, excluding points of branching [12]. LM was measured at its mid-point and the LAD/ RCA was measured 0.2 to $0.5 \mathrm{~mm}$ from its origin [3]. Measurements and imaging were performed by three experienced operators (SM, JS and KB, all with $>5$ years experience in coronary echocardiography in congenital heart disease).

Current echocardiographic data does not specify the point of the cardiac cycle (systole or diastole) over which measurements should be taken [3]. In addition, practically, it is often difficult to get images in both phases. Therefore, only one measurement was taken for each coronary measurement but it was also recorded as to which phase the measurement was taken, in order to compare it fairly to MRI. The number of proximal LAD branches imaged was also recorded in order to give an estimate of distal coronary tree visualization.

\section{Statistical methods}

First, bland-Altman plots were used to assess agreement between MRI and echocardiography for coronary dimensions. Using information gleaned from this analysis, regression analyses were planned in order to describe the relationship between MRI and echocardiographic measurements.

Distal coronary visualization, assessed by number of LAD branches visualized, was compared between modalities using Wilcoxon Signed Ranks test.

A repeated measures analysis of variance models for coronary dimensions was constructed in order to assess if the coronary distensibility in children results in different dimensions according to cardiac phase. A repeated measures model was used in order to account for within-subject and between-subject variability arising from testing multiple segments over two phases in each patient. Assumptions of sphericity were formally tested, and accepted, using Mauchly's test. The Bonferroni correction is used to correct for multiple comparisons.

Statistical analyses were performed on SPSS (version 19) 2010. Variables are described using mean \pm standard deviation (s.d.).

\section{Results}

Subjects

Fifty children participated in this study prospectively with a mean age of $4 \pm 4.4$ years (range 18 days to 18 years). This included 24 boys (mean age $4.5 \pm$ 4.6 years) and 26 girls (mean age $3.6 \pm 4.3$ years; no significant age difference according to sex, $p=0.46$ by independent samples $t$-test). Mean weight was $15.5 \pm 11.6 \mathrm{~kg}$ (range 3.7 to $52 \mathrm{~kg}$ ) and mean height was $94 \pm 29 \mathrm{~cm} .48$ children underwent imaging for follow-up of congenital heart disease, 1 child for cardiomyopathy and 1 for resolved Kawasaki disease.

\section{Reference values}

Bland-Altman plots (Figs. 1, 2 and 3) show a systematic bias between echocardiographic and MRI measurements for all coronary measurements (i.e., as the coronary

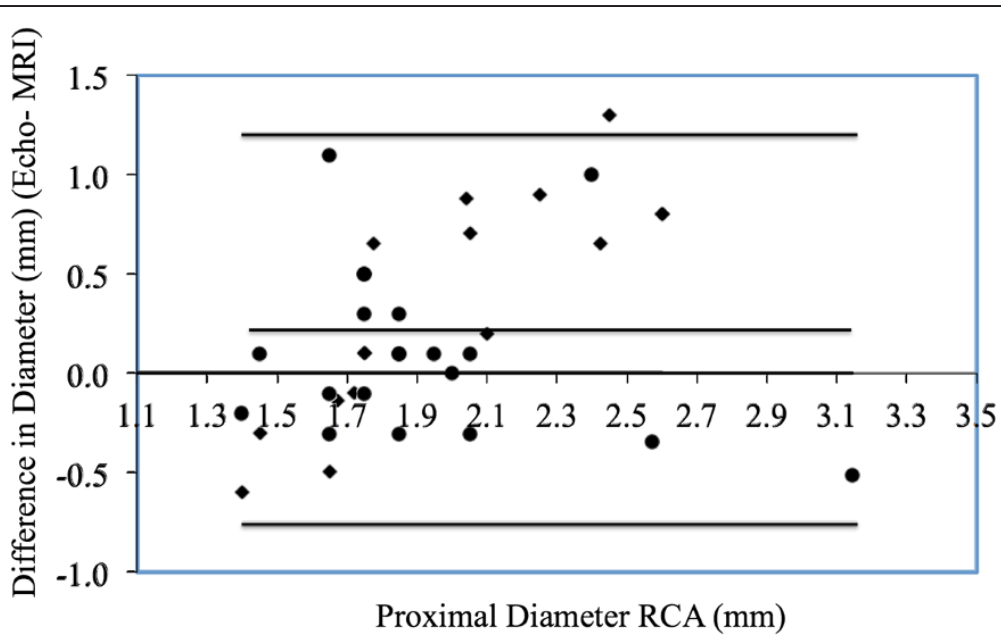

Fig. 1 Bland Altman Mean vs. Difference plots for Echo and MRI RCA measurements. • Diastolic measurements. $\bullet$ Systolic measurements 


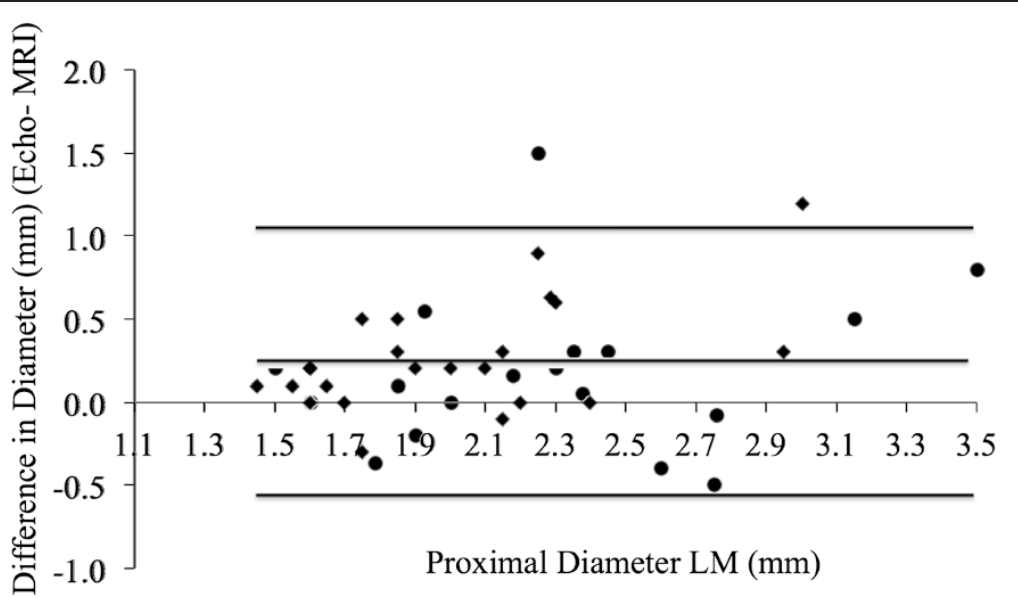

Fig. 2 Bland Altman Mean vs. Difference plots for Echo and MRI LM measurements. • Diastolic measurements. $\bullet$ Systolic measurements

artery gets larger, the greater the discrepancy between echocardiography and MRI).

However, this may be more usefully summarized by ignoring the segment imaged, removing outliers and pooling data. If this is done we get a simple message: there is indeed a systematic bias for CMRA dimensions between 1 and $2.5 \mathrm{~mm}$. This is demonstrated on the Bland-Altman plot (Fig. 4). More importantly, the accuracy between echo and MRI measurements is not clinically acceptable. The scatter clearly shows that, at dimensions between 1.3 to $2.5 \mathrm{~mm}$, the spread of agreement is more than $0.5 \mathrm{~mm}$ at all points. Hence no further analysis was made to describe the correlation (i.e., regression analysis was not felt appropriate due to poor agreement).

\section{CMRA evaluation}

Operator certainty regarding the origin of the three main coronary arteries (RCA, LAD and left circumflex (LCx)) was achieved in 47 out of 50 cases for MRI. The three uncertain cases were infant below a year of age (3, 9 and 11 months) and with heart rates $>100 \mathrm{bpm}$. In comparison, certainty was achieved in 41 out of 50 cases for echo. The nine uncertain cases were older patients (mean 4.8 years, range 9 months to 18 years). In two cases, MRI detected abnormalities in coronary origin classified as normal with certainty on echocardiography. These two cases had confirmed abnormalities on surgery. Both were slightly older children (13 months and 8 years age respectively). The 13-month-old child had an anomalous origin of the left circumflex origin from the right coronary artery. The 8-year old child had ventriculo-arterial discordance and had a single coronary artery from left-hand sinus two (Fig. 6).

In total, there were 6 abnormal coronary origins. 5 out of 6 were identified by MRI and the remaining one was classified as unsure. 4 out of 6 were correctly identified on echocardiogram with the remaining two, incorrectly classified as normal as described above. (Sample images: Figs. 5, 6 and 7).

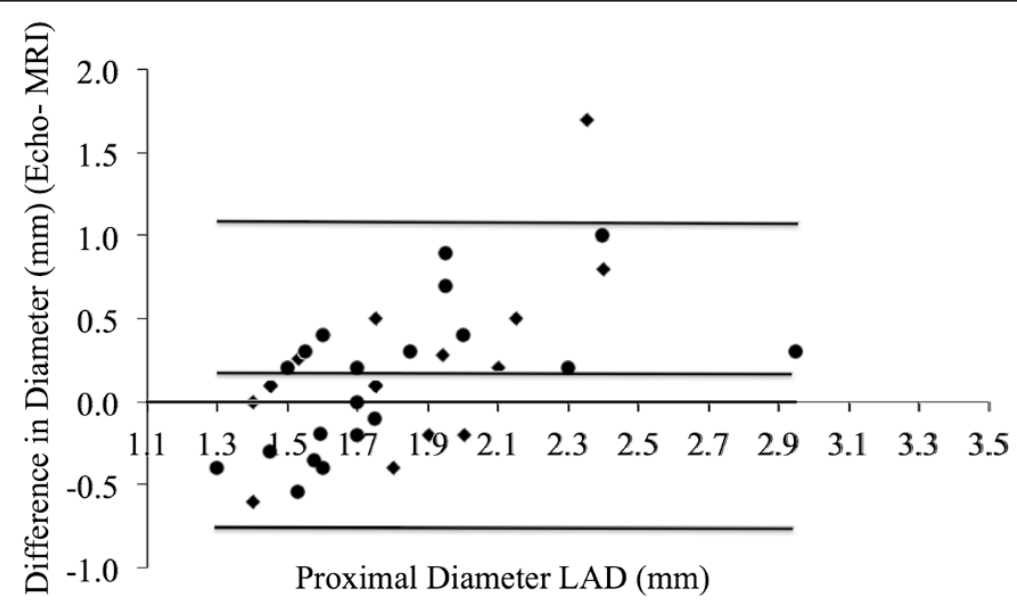

Fig. 3 Bland Altman Mean vs. Difference plots for Echo and MRI LAD measurements. $\bullet$ Diastolic measurements. Systolic measurements 


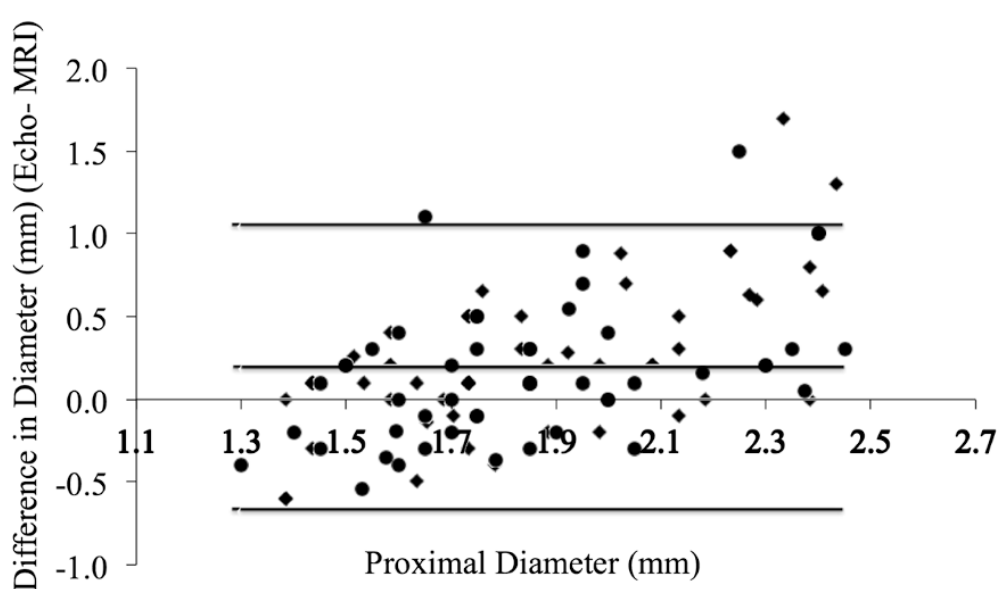

Fig. 4 Bland Altman Mean vs. Difference plots for all Echo and MRI measurements with outliers excluded. • Diastolic measurements. Systolic measurements

Overall, CMRA performed better than echocardiography in terms of distal visualization of the coronary tree, as defined by number of proximal LAD branches imaged ( $p=0.001$ by Wilcoxon Signed Ranks test). The median number of LAD branches on MRI was 1 (interquartile range 0 to 2 ) and on echo was 0 (interquartile range 0 to 1 ).

CMRA measurements did vary according to cardiac phase. The repeated measures model demonstrates that the systolic coronary dimension (estimated marginal mean $1.90 \pm 0.05 \mathrm{~mm}$ ) is greater than the diastolic measurement (estimated marginal mean $1.84 \pm 0.05 \mathrm{~mm}(p=0.002)$.

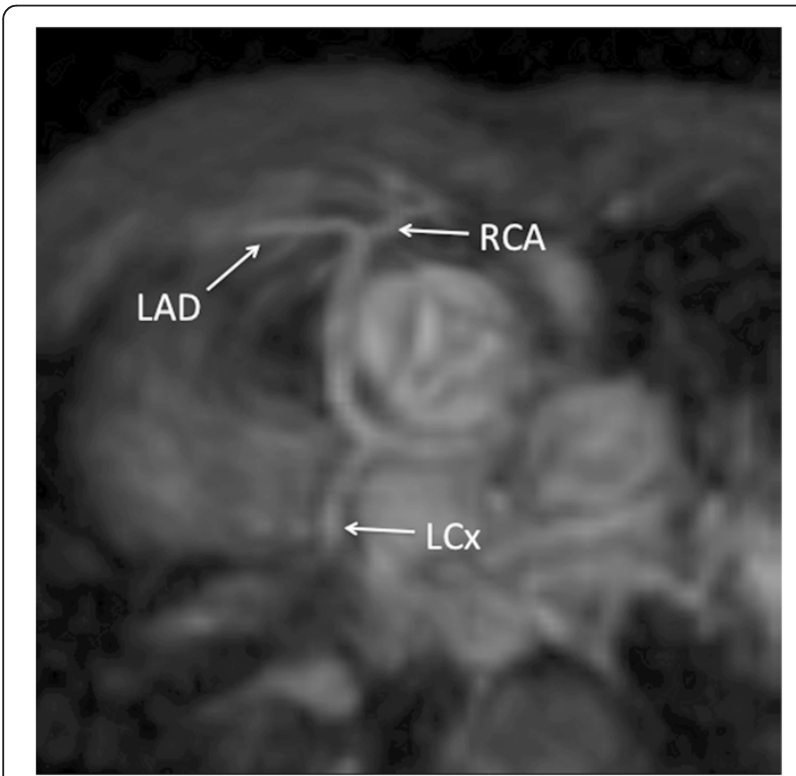

Fig. 5 3-year old girl with Dextrocardia and Tricuspid Atresia. Single Coronary artery imaged with MRA in systole. Echocardiographic data corresponded with MRA findings

\section{Discussion}

This study has important implications for the follow-up of Kawasaki disease in particular. MRI is particularly useful for follow-up of Kawasaki Disease and Coronary Allograft Vasculopathy as anatomic evaluation can be combined with functional evaluation with pharmacological stress testing. Intra- and inter-observer error in coronary measurements for both echocardiography and CMRA have been previously separately investigated and shown to be low for both modalities $[3,7]$. However, this

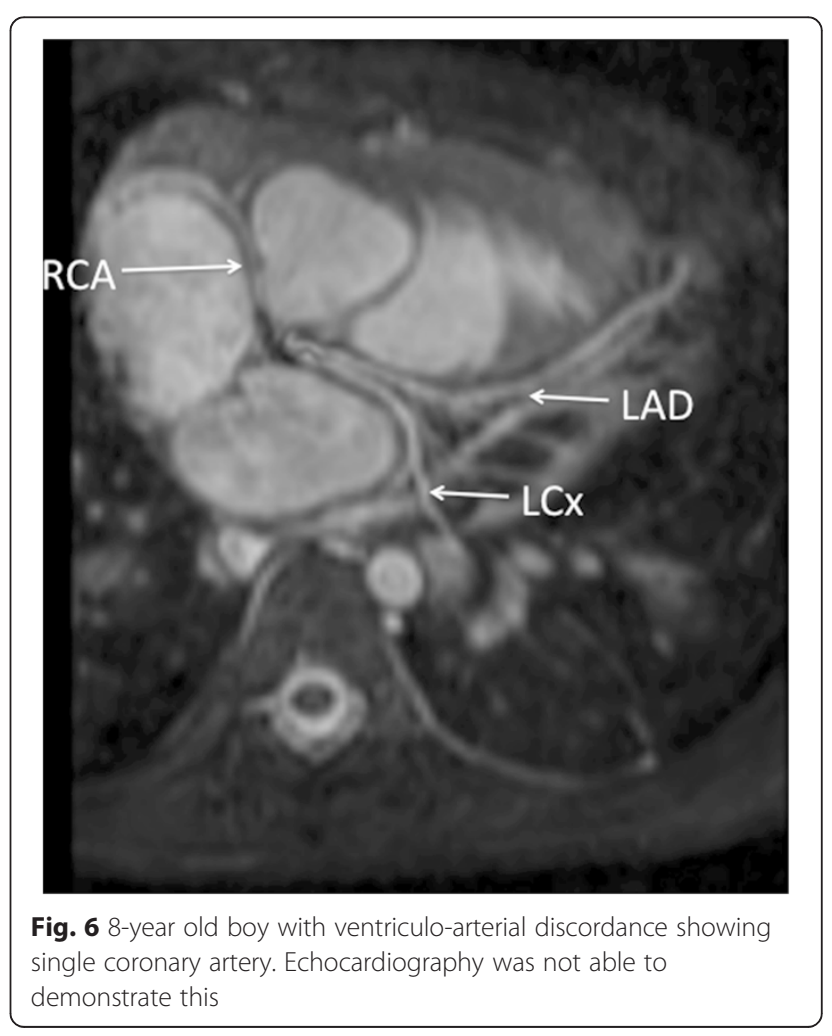




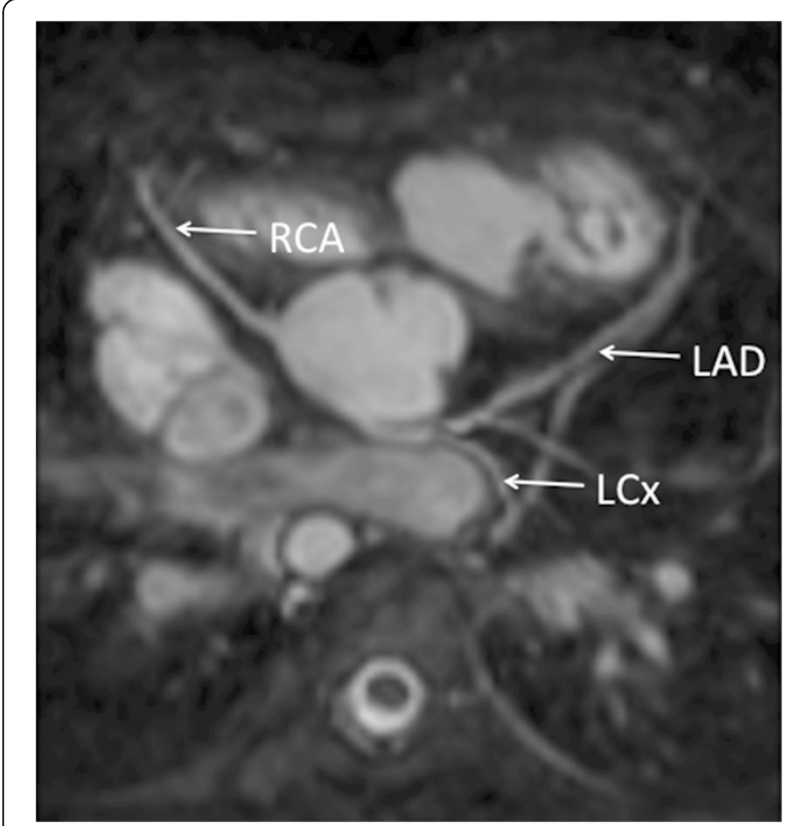

Fig. 7 7-year old girl with repaired pulmonary atresia and ventricular septal defect. RCA arises from posterior non-coronary cusp. Findings were confirmed by echocardiography

study shows poor agreement between techniques. This may be in part, due to the current difficulty achieving sub-millimeter acquired resolution for coronary MRA in small children. The study resolution of $1-1.5 \mathrm{~mm}^{3}$ acquired $\left(0.5-0.75 \mathrm{~mm}^{3}\right.$ reconstructed) was chosen in order to give the optimum image quality as further increase in resolution would cause reduction in signal-tonoise ratio and would result in sub-optimal image quality. Improvements in image quality in infants should be the subject of future studies. It is hoped that, in the future, specific normative coronary dimensions for CMRA are available. The difficulty imaging normal volunteers in this age group has precluded this to date. Hence, until such data becomes available, the utility of coronary measurement below $3 \mathrm{~mm}$ lies in serial follow-up rather than for normative scoring. In particular, when reporting MRI coronary dimensions, we should not use published z-score values for echocardiography [3]. In the past, clinicians have merely assumed that dimensions were normal on CMRA if they equated to normal published echocardiographic z-scores. This study shows that this assumption is erroneous. One may argue that this result was to be expected because echocardiography relies on high contrast in the vessel wall and low contrast in the lumen, whereas CMRA is the opposite. Although 'blackblood' coronary MRI exists [13] and contrast-enhanced echocardiography exists, the purpose of this study was to evaluate the commonly used clinical echocardiographic and MRI techniques.
In keeping with previous studies, this study shows an excellent (94\%) success rate for CMRA detection of coronary origins in children $[7,14,15]$. Dual-phase imaging may be advantageous as it has been previously shown that image quality of each proximal coronary segment can vary unpredictably within the same patient according to selection of the systolic or diastolic rest period for imaging [7]. Infants with high heart rates remain challenging [14] and echocardiography remains the modality of choice for this age group. Naturally, echocardiography remains the first choice for cardiac imaging in all children due to the ease of application and the acceptability from a child's perspective. Success rates are difficult to report from literature, as the technique is operator dependent with some operators showing excellent accuracy [16-18]. Success rates are even higher in newborn infants [19]. Nevertheless, there remains an important false negative reporting rate, which is difficult to quantify [20]. Given the operator-dependency, any doubt regarding coronary anomaly with potentially malignant course, should have further imaging [16]. Our study also shows that distal coronary tree imaging in children is superior with CMRA in comparison to echocardiography. This is another useful point of note from this study for clinical follow up of coronary disease in children. Multidetector CT angiography has excellent success rates in determining coronary origins and is becoming routinely used clinically to detect coronary stenosis [21, 22]. However, it does involve potentially harmful radiation use in young children.

Unfortunately, coronary distensibility in children has been largely neglected in the published literature $[2,3$, 23]. Although an important recognized variable in adults, [24] this factor has been ignored when generating echocardiographic normal values for coronary dimensions in children [2, 3, 23]. The given reference study [3] was chosen specifically as the details regarding how measurements are to be taken are most precise. Even this study neglects to mention whether values are to be taken in systole or diastole. From clinical experience using echocardiography, it is usually difficult to get clear pictures for each segment in both phases and so it is understandable that this point is not labored. However, our data from the dual-phase CMRA measurements shows that dimensions do indeed vary according to phase. Future normal values for coronary dimensions in children should note this finding and provide phasespecific references.

\section{Conclusions}

This study shows that echocardiographic reference values should not be applied to CMRA measurements of coronary dimensions. Looking forward, the development of CMRA-specific reference values would be helpful, 
although such normative data is difficult to acquire in children. Our study confirms the perception that CMRA remains extremely successful in the identification of coronary origins in children but echocardiography remains superior in small infants. Our study also shows that CMRA is superior to echocardiography in the imaging of the distal coronary tree of children. Finally, the study also demonstrates the need to establish heart phasespecific coronary reference ranges.

\section{Competing interests}

The authors declare that they have no competing interests.

\section{Authors' contributions}

TH, SM, SP, IV, KB, JS, GG: Planning, data acquisition, data analysis and drafting manuscript. MH, RB: Planning, data analysis and drafting manuscript. All authors read and approved the final manuscript.

\section{Acknowledgements}

The authors acknowledge financial support from the Department of Health via the National Institute for Health Research (NIHR) comprehensive Biomedical Research Centre award to Guy's \& St Thomas' NHS Foundation Trust in partnership with King's College London and King's College Hospital NHS Foundation Trust".

Dr Gerald Greil's department is part of the Centre of Excellence in Medical Engineering is funded by the Wellcome Trust and EPSRC under grant number WT 088641/Z/09/Z".

King's College London is a British Heart Foundation centre of excellence funded by the British Heart Foundation award RE/08/003.

\section{Author details}

${ }^{1}$ Division of Imaging Sciences, King's College London, NIHR Biomedical Research Centre at Guy's \& St Thomas' NHS Foundation Trust, London, UK. 'Department of Paediatric Cardiology, Evelina London Children's Hospital, Guy's \& St Thomas' NHS Foundation Trust, Westminster Bridge Road, London, UK. ${ }^{3}$ Department of Pediatrics, UT Southwestern Medical Center, Children's Medical Center, 1935 Medical District Drive, Dallas, TX, USA.

\section{Received: 24 October 2014 Accepted: 22 October 2015}

Published online: 27 October 2015

\section{References}

1. Geva T, Kreutzer J. Diagnostic pathways for evaluation of congenital heart disease. In: Crawford MH, DiMarco JP, editors. Cardiology. London: Mosby International; 2001. p. 7-41.

2. McCrindle BW, Li JS, Minich LL, Colan SD, Atz AM, Takahashi M, et al. Coronary artery involvement in children with Kawasaki disease: risk factors from analysis of serial normalized measurements. Circulation. 2007;116(2):174-9.

3. Olivieri L, Arling B, Friberg M, Sable C. Coronary artery Z score regression equations and calculators derived from a large heterogeneous population of children undergoing echocardiography. J Am Soc Echocardiogr. 2009;22(2):159-64.

4. Greil GF, Stuber M, Botnar RM, Kissinger KV, Geva T, Newburger JW, et al. Coronary magnetic resonance angiography in adolescents and young adults with kawasaki disease. Circulation. 2002;105(8):908-11.

5. Uribe S, Hussain T, Valverde I, Tejos C, Irarrazaval P, Fava M, et al. Congenital Heart Disease in Children: Coronary MR Angiography during Systole and Diastole with Dual Cardiac Phase Whole-Heart Imaging. Radiology. 2011;260(1):232-40.

6. Beerbaum P, Sarikouch S, Laser KT, Greil G, Burchert W, Korperich H. Coronary anomalies assessed by whole-heart isotropic 3D magnetic resonance imaging for cardiac morphology in congenital heart disease. J Magn Reson Imaging. 2009;29(2):320-7.

7. Hussain T, Lossnitzer D, Bellsham-Revell H, Valverde I, Beerbaum P, Razavi R, et al. Three-dimensional dual-phase whole-heart MR imaging: clinical implications for congenital heart disease. Radiology. 2012;263(2):547-554.
8. Uribe S, Tangchaoren T, Parish V, Wolf I, Razavi R, Greil G, et al. Volumetric Cardiac Quantification by Using 3D Dual-Phase Whole-Heart MR Imaging. Radiology. 2008;248(2):606-14.

9. Pruessmann KP, Weiger M, Scheidegger MB, Boesiger P. SENSE: sensitivity encoding for fast MRI. Magn Reson Med. 1999;42(5):952-62.

10. Ustun A, Desai M, Abd-Elmoniem KZ, Schar M, Stuber M. Automated identification of minimal myocardial motion for improved image quality on MR angiography at 3 T. AJR Am J Roentgenol. 2007;188(3):W283-90.

11. Kim WY, Stuber M, Kissinger KV, Andersen NT, Manning WJ, Botnar RM. Impact of bulk cardiac motion on right coronary MR angiography and vessel wall imaging. J Magn Reson Imaging. 2001;14(4):383-90.

12. Newburger JW, Takahashi M, Gerber MA, Gewitz MH, Tani LY, Burns JC, et al. Diagnosis, treatment, and long-term management of Kawasaki disease: a statement for health professionals from the Committee on Rheumatic Fever, Endocarditis and Kawasaki Disease, Council on Cardiovascular Disease in the Young. Am Heart Assoc Circ. 2004;110(17):2747-71.

13. Botnar RM, Kim WY, Bornert P, Stuber M, Spuentrup E, Manning WJ. 3D coronary vessel wall imaging utilizing a local inversion technique with spiral image acquisition. Magn Reson Med. 2001;46(5):848-54.

14. Tangcharoen T, Bell A, Hegde S, Hussain T, Beerbaum P, Schaeffter T, et al. Detection of coronary artery anomalies in infants and young children with congenital heart disease by using MR imaging. Radiology. 2011;259(1):2407.

15. Su JT, Chung T, Muthupillai R, Pignatelli RH, Kung GC, Diaz LK, et al. Usefulness of real-time navigator magnetic resonance imaging for evaluating coronary artery origins in pediatric patients. Am J Cardiol. 2005;95(5):679-82.

16. Attili A, Hensley AK, Jones FD, Grabham J, DiSessa TG. Echocardiography and coronary $\mathrm{CT}$ angiography imaging of variations in coronary anatomy and coronary abnormalities in athletic children: detection of coronary abnormalities that create a risk for sudden death. Echocardiography. 2013;30(2):225-33.

17. Frommelt PC, Berger S, Pelech AN, Bergstrom S, Williamson JG. Prospective identification of anomalous origin of left coronary artery from the right sinus of valsalva using transthoracic echocardiography: importance of color Doppler flow mapping. Pediatr Cardiol. 2001;22(4):327-32.

18. Frommelt PC, Friedberg DZ, Frommelt MA, Williamson JG. Anomalous origin of the right coronary artery from the left sinus of valsalva: transthoracic echocardiographic diagnosis. J Am Soc Echocardiogr. 1999;12(3):221-4.

19. Pasquini L, Sanders SP, Parness IA, Wernovsky G, Mayer Jr JE, Van der Velde $\mathrm{ME}$, et al. Coronary echocardiography in 406 patients with d-loop transposition of the great arteries. J Am Coll Cardiol. 1994;24(3):763-8.

20. Pelliccia A, Spataro A, Maron BJ. Prospective echocardiographic screening for coronary artery anomalies in 1,360 elite competitive athletes. Am J Cardiol. 1993;72(12):978-9.

21. Vanhoenacker PK, Heijenbrok-Kal MH, Van Heste R, Decramer I, Van Hoe LR, Wijns $W$, et al. Diagnostic performance of multidetector $C T$ angiography for assessment of coronary artery disease: meta-analysis. Radiology. 2007;244(2):419-28.

22. Ghadri JR, Kazakauskaite E, Braunschweig S, Burger IA, Frank M, Fiechter M, et al. Congenital coronary anomalies detected by coronary computed tomography compared to invasive coronary angiography. BMC Cardiovasc Disord. 2014;14:81.

23. Dallaire F, Dahdah N. New equations and a critical appraisal of coronary artery Z scores in healthy children. J Am Soc Echocardiogr. 2011;24(1):60-74.

24. Lin K, Lloyd-Jones DM, Liu Y, Bi X, Li D, Carr JC. Noninvasive Evaluation of Coronary Distensibility in Older Adults: A Feasibility Study with MR Angiography. Radiology. 2011;261(3):771-8. 